\title{
Angle insensitive enhancement of organic solar cells using metallic gratings
}

\author{
Aimi Abass ${ }^{1 *}$ and Honghui Shen ${ }^{2}$, Peter Bienstman ${ }^{2}$, Bjorn Maes ${ }^{3}$ \\ ${ }^{1}$ Department of Electronic and Information Systems (ELIS), \\ Ghent University, Sint-Pietersnieuwstraat 41, B-9000 Gent, Belgium \\ ${ }^{2}$ Photonics Research Group (INTEC), Ghent University - imec, \\ Sint-Pietersnieuwstraat 41, B-9000 Ghent, Belgium and \\ ${ }^{3}$ Micro- and Nanophotonic Materials Group, \\ Institut de Physique, University of Mons, \\ 20 Place du Parc, B-7000 Mons, Belgium
}

(Dated: January 10, 2011)

\begin{abstract}
We explore the optical enhancement of organic photovoltaic cells by incorporating a metallic grating as the back contact. We numerically demonstrate a strongly enhanced light absorption exploiting a complex interplay between multiple electromagnetic wave phenomena, among which Surface Plasmon Polariton (SPP) resonances, waveguide mode resonances, Fabry-Perot modes and scattering. We focus on a triangular grating structure and describe the particular opportunities to obtain a good angular performance. In addition we introduce a novel multiperiodic geometry that incorporates multiple types of SPP resonances. Our triangular structure shows an increased absorption of $15.6 \%$ with the AM1.5G spectrum in the $300-800 \mathrm{~nm}$ wavelength range. For the multiperiodic grating case a significant further increase to $20.7 \%$ is shown.
\end{abstract}

*Electronic address: aimi.abass@elis.ugent. be 


\section{INTRODUCTION}

Nowadays, organic solar cells are still restricted to thin films on the order of $200 \mathrm{~nm}$ for optimal electronic properties [1], which makes efficient photon management an important issue [2-4]. Indeed the typical absorption spectrum of polymer films has room for improvement, usually in the infrared range. In addition, even thinner active layers, on the order of $100 \mathrm{~nm}$, would be interesting as this leads to better electrical properties.

An interesting novel technique to boost the absorption of thin film solar cells, involves the use of metallic structures with Surface Plasmon Polariton (SPP) resonances [5]. These modes can achieve a strong confinement and scattering of the incoming light. In addition one can engineer the modes so that the absorption in the metal remains limited. Several metal geometries (gratings, particles [6-9]) and their locations in the cell (front, middle or back) are under active investigation for different types of solar cells. Plasmonic grating structures [5, 10-15] form a particularly interesting class, as they involve the patterning of an available metallic electrode.

In this work, we focus on triangular grating structures for the metallic back contact (Fig. 1). These type of gratings are less studied than the square gratings, however we will show that the usage of these triangular gratings can give great absorption enhancement. Several lithographic methods (nanoimprint, optical or electron beam lithography) for metallic grating structures can be used to produce these types of gratings [16-19]. The chosen metal back contact material is silver, as it allows for low metal absorption losses, and beneficial SPP resonance wavelengths. An example of the usage of silver square gratings to enhance silicon thin film solar cells is shown in recent publications $[12,15]$. Here, we explore the characteristics further and we examine specifically the angular behaviour of the excited modes for bulk heterojunction solar cells made of P3HT:PCBM (poly(3-hexylthiophene):[6,6]-phenylC61-butyric acid methyl ester) blends. The depth of the metallic grating is found to be crucial for a good wide-angle response for the case of normal triangular gratings. We show that an even greater absorption enhancement is achieved through more complex triangular gratings. We also investigate the use of $2 \mathrm{D}$ pyramidal gratings. The design strategies we develop here are applicable to various cell materials.

Section II describes the numerical procedure and the initial strategy for boosting the absorption. Section III is the main part and discusses the use of various resonances in our 


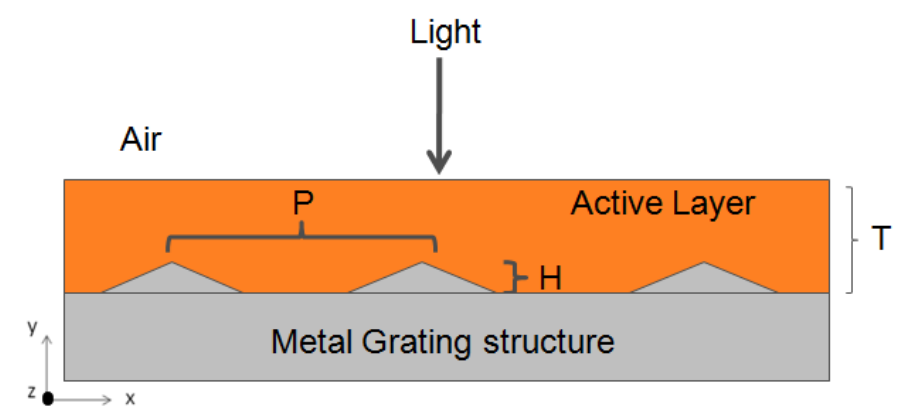

FIG. 1: The studied solar cell system, consisting of air, polymer active layer and metallic grating. $\mathrm{T}$ is the (maximum) polymer thickness, $\mathrm{H}$ the triangle height and $\mathrm{P}$ the grating period.

devices.

\section{METHOD}

We simulate incoming light interaction with our solar cell using the finite element method, as implemented in the COMSOL multiphysics software package. The bulk heterojunction P3HT:PCBM polymer is considered as a homogeneous material with a dispersive refractive index. Our refractive index data is obtained from ellipsometry measurements done at imec (Interuniversity Microelectronics Center, Leuven, Belgium). The refractive index data of the P3HT:PCBM blend is shown in [6]. Due to the periodic nature of the problem, only one period needs to be simulated by using the proper boundary conditions. In order to study angular dependence, we employ Floquet-Bloch conditions on the cell boundaries [20]. The top and bottom part of the computational domain is terminated with absorbing Perfectly Matched Layer regions.

We are particularly interested in the absorption enhancement within the polymer itself. To quantify this we integrate the divergence of the Poynting vector $\vec{S}$ within the polymer region only and normalize it to the incoming power over one period $P_{i n c}$ :

$$
A(\lambda)=\frac{\int(\nabla \cdot \vec{S}) d V_{\text {polymer }}}{P_{\text {inc }}}
$$

To quantify the absorption capability of our solar cell structure in the wavelength region of 
interest (300-800nm), we define a normalized weighted integrated absorption for the AM1.5G spectrum:

$$
A_{\text {int }}=\frac{\int\left(P_{A M 1.5 G}(\lambda) \times A(\lambda)\right) d \lambda}{\int P_{A M 1.5 G}(\lambda) d \lambda} .
$$

The choice of the wavelength region is connected to the P3HT:PCBM polymer blend absorption properties. For this material, we know that charge creation through photon absorption is still possible even up to the wavelength of $800 \mathrm{~nm}[21]$.

Our approach in optimizing the triangular gratings starts from looking at planar solar cell structures. $A_{i n t}$ as a function of film thickness for normal incidence is shown in Fig. 2(a). The oscillation is due to interference or Fabry-Perot effects in the active layer. Several absorption spectra are shown in Fig. 2(b). The planar data provides us with approximative guidelines for the grating structure. First, the effective thickness decreases when introducing triangles, thus to obtain an approximate, optimum Fabry-Perot condition with the grating, we are guided to choose $\mathrm{T}$ in the range $60-150 \mathrm{~nm}$ or in the range $200-250 \mathrm{~nm}$. This will bring the effective thickness closer the the first or second Fabry-Perot maximum, respectively. Second, the absorption spectra (Fig. 2(b)) indicate where we should position extra resonances. Indeed, between 620 and $800 \mathrm{~nm}$ the absorption is limited so a boost is needed.

\section{ENHANCEMENT TECHNIQUES}

We present several plasmonic grating enhancement techniques, spread over four categories. Section III A looks into the use of triangular grating SPP modes. Section IIIB employs polymer waveguide modes in thicker active layers. Section III C discusses a more complex grating device to access strongly localized SPP modes. Finally, section III D shows results on pyramidal gratings to obtain a polarization independent absorption enhancement.

\section{A. Using SPP modes}

Here we show how one can strategically place plasmon resonances in order to achieve an angle independent enhancement. The resonant wavelengths of the modes depend strongly on the geometry of the grating. The grating periodicity induces folding of the band structure which determines strongly where the resonances are located $[22,23]$. Full-field mode-solvers 
(a)

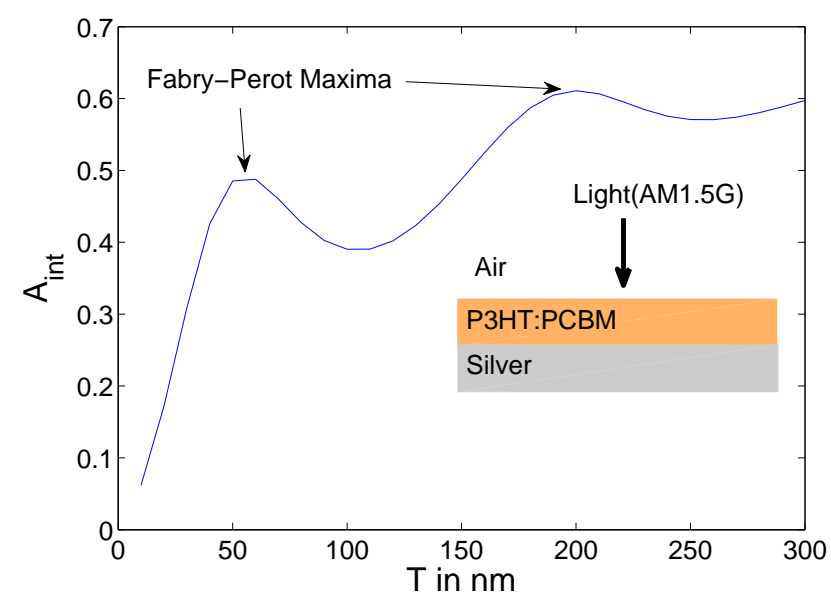

(b)

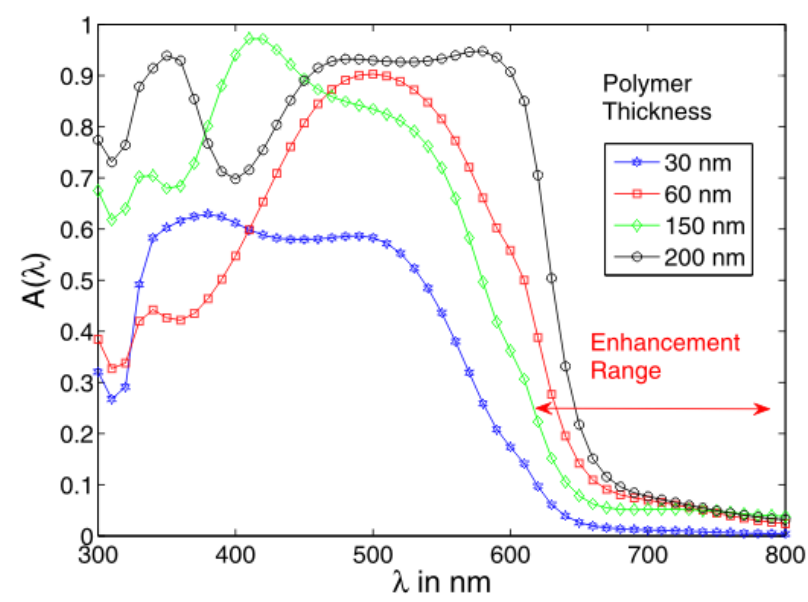

FIG. 2: Calculations for planar structures (see inset). (a) Integrated absorption $A_{\text {int }}$ as a function of film thickness ( $\mathrm{T}$ ) for normal incidence. (b) Absorption spectrum $A(\lambda)$ in the polymer for different thicknesses T.

can be employed to find the bands, but in the case of shallow gratings we obtain a good estimate simply by folding the dispersion of the flat interface (silver-polymer). The folding procedure is performed in Fig. 3(a) with a periodicity $\mathrm{P}=300 \mathrm{~nm}$.

This dispersion folding procedure should be compared with the rigorous finite element simulations in Fig. 3(b) and (c), where we show the absorption spectrum at different angles of incidence for a shallow grating structure with thickness $\mathrm{T}=150 \mathrm{~nm}$, period $\mathrm{P}=300 \mathrm{~nm}$ and height $\mathrm{H}=60 \mathrm{~nm}$. The absorption spectra in this section are for TM polarization (meaning the magnetic field only has a component in the $\mathrm{z}$ direction of Fig. 1), unless mentioned 
(a)

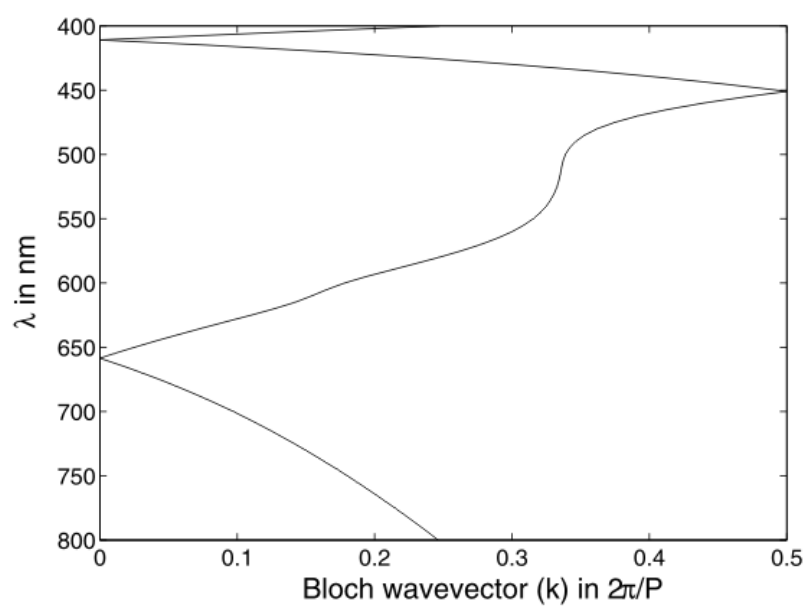

(b)

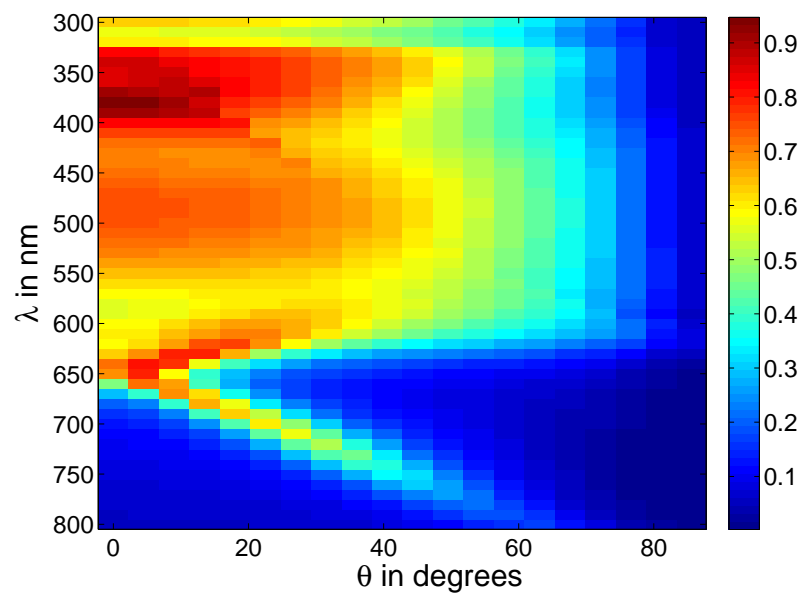

(c)

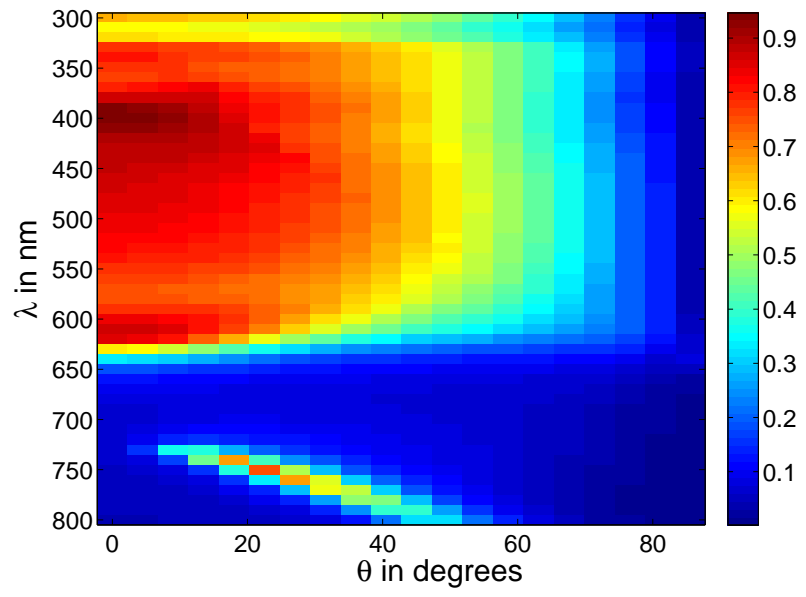

FIG. 3: (a) Folded band diagram of SPP mode on planar silver-polymer interface for $\mathrm{P}=300 \mathrm{~nm}$.

(b) Absorption spectrum $A(\lambda)$ at different angles for $\mathrm{FF}=1$ ( $\mathrm{T}=150 \mathrm{~nm}, \mathrm{P}=300 \mathrm{~nm}, \mathrm{H}=60 \mathrm{~nm})$. (c) $A(\lambda)$ at different angles for $\mathrm{FF}=0.5$. 
otherwise. Fig. 3(b) has fill-factor $\mathrm{FF}=1$ (base length of triangle divided by the period). A clear correspondence is noticed between the crossings around 660nm in Fig. 3(a) and Fig. 3(b).

The structure of Fig. 3(c), which has $F F=0.5$, shows a significantly different dispersion. There is a strong splitting of the modes but the central wavelength is still around 660nm. In addition, one of the modes (the 'bright' mode) can be excited at normal incidence, while the other mode (the 'dark' mode) needs an angle $\theta \neq 0$. This character is crucial for angle-dependent optimization in solar cells.

These aspects are explained by the field profiles of the modes at $k=0$ obtained via eigenmode analysis also by COMSOL, see Fig. 4. The eigenmode analysis was done to obtain the dark mode field profile which is unexcitable by plane wave incidence. We notice that the modes are lowest order, corresponding indeed with the lowest frequency crossing at Bloch wavevector $k=0$. First of all the difference between the field profiles leads to different field energies or frequencies, lifting the degeneracy. Second, because of symmetry the total dipole moment of the low frequency mode is zero, leading to the dark character, as the overlap with a perpendicular plane wave is zero.

The splitting is highly dependent on the grating geometry, more specifically on the fillfactor FF and triangle height $\mathrm{H}$. The effects of the two geometrical parameters can be traced by examining the Fourier expansion of the grating profile [24]. It can be shown that at $\mathrm{FF}=1$ (Fig. 3(b)), the second harmonic of the profile Fourier expansion is 0 leading to less splitting of the first order crossing at $k=0$. In the case shown in figure 3 , the dark mode has a lower energy than the bright mode and thus appears at a longer wavelength. Depending on the structure in question, the vice versa situation is also possible [23].

As pointed out $[24,25]$, increasing the grating height leads to a flatter SPP dispersion. Indeed, by increasing the grating height we get a more localized behaviour of the resonances instead of a propagating behaviour. This works well to our advantage in a solar cell design, since we would like to use a resonance that is not angle-dependent. We demonstrate this effect by increasing $\mathrm{H}$ in our structure (Fig. 5). Here, for clarity we show the absorption in the metal, instead of in the polymer. The splitting of the dark and bright mode also increases when the grating deepens, as this enhances the difference between the mode field profiles.

Thus, with the proper parameter choice we can utilize both dark and bright modes 


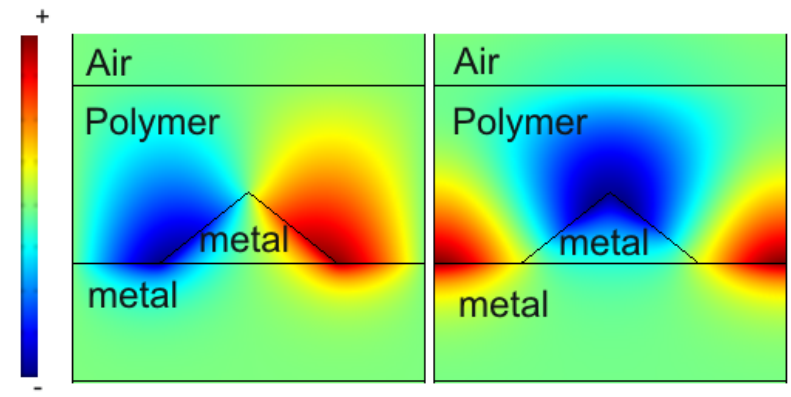

FIG. 4: Magnetic field $\left(H_{z}\right)$ profile at $\mathrm{k}=0$ of dark mode (left, $\lambda=727 \mathrm{~nm}$ ) and bright mode (right, at $\lambda=615 \mathrm{~nm})$. Same geometrical parameters as in Fig.3(c).
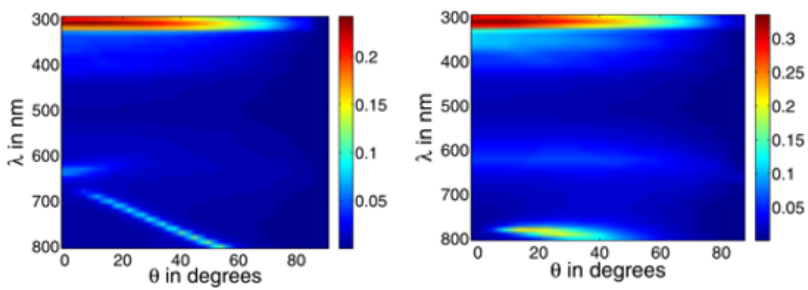

FIG. 5: The effect of triangle height $\mathrm{H}$ on the metal absorption spectrum for a grating with $\mathrm{FF}=0.5$, $\mathrm{P}=300 \mathrm{~nm}, \mathrm{~T}=150 \mathrm{~nm}$, and $\mathrm{H}=25 \mathrm{~nm}$ (left) or $\mathrm{H}=100 \mathrm{~nm}$ (right), respectively.

simultaneously for non-perpendicular incidence. In Fig. 6(a) and (b) two SPP peaks at $600 \mathrm{~nm}$ and $650 \mathrm{~nm}$ clearly boost the polymer absorption. The optimum incidence angle here is $\theta=13.5^{\circ}$. These simulation results (Fig. 6(b)) show an absorption enhancement up to 7 times due to the dark mode at $650 \mathrm{~nm}$, from below $10 \%$ to nearly $70 \%$. This clearly suggests that most of the SPP resonance absorption occurs in the polymer, instead of in the metal. It corresponds with the SPP mode field profile being well distributed in the polymer (inset in Fig. 6(b)). Moreover, as the field is not concentrated near the metal interface, possible losses due to exciton quenching near metal surfaces should be reduced [26]. Importantly, with the chosen geometric parameters, the absorption in the wavelength range $<550 \mathrm{~nm}$ remains largely intact.

With larger periods, higher order plasmonic modes can be accessed to boost absorption. In that case the first Brillouin zone becomes narrower and higher order modes can exist in the wavelength region of interest. With larger periods it is also possible to have a diffraction edge in the neighborhood of our enhancement range. However, for our structures the SPP 
(a)

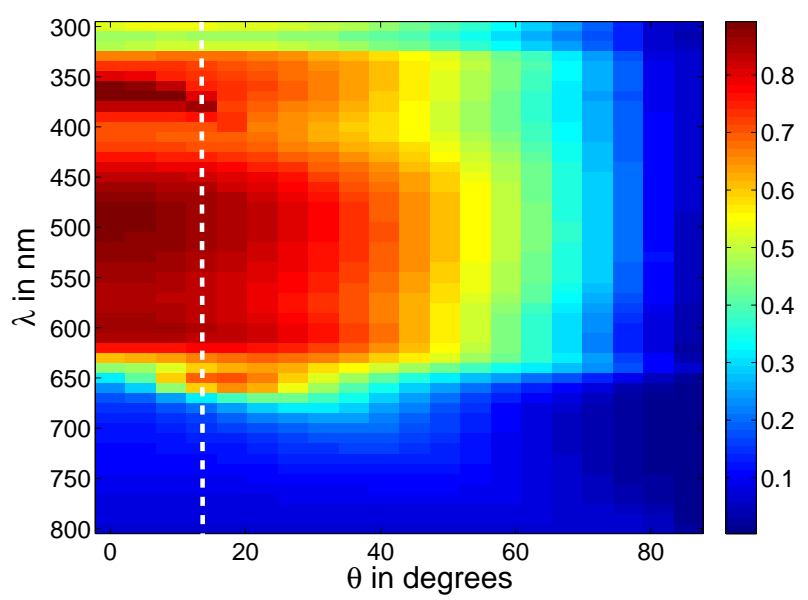

(b)

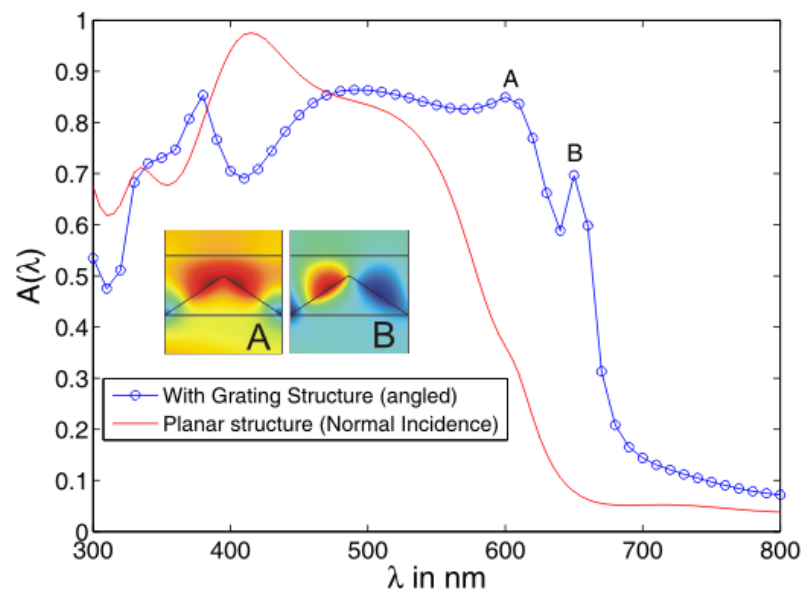

FIG. 6: Angularly optimized grating with $\mathrm{T}=150 \mathrm{~nm}, \mathrm{P}=300 \mathrm{~nm}, \mathrm{H}=100 \mathrm{~nm}, \mathrm{FF}=1$. (a) $A(\lambda)$ versus angle of incidence. The white, dashed line indicates $\theta=13.5^{\circ}$. (b) $A(\lambda)$ at $\theta=13.5^{\circ}$ (line-circles) compared to a planar structure at normal incidence (line). The insets show the magnetic field profile at the two peaks indicated with $\mathrm{A}$ and $\mathrm{B}$.

resonances seemed to offer better enhancement. For larger periods with strong interaction between SPP resonances and diffraction, such as in lattice resonances [27], further studies can be performed. Fig. 7(a) shows the absorption spectrum at 500nm grating periodicity for normal incident light. The resonance peaks of the second order are also predicted quite well with simple band folding. Fig. 7(b) shows the magnetic field profile at the two peaks indicated in (a). As would be expected of higher order modes, the field profile of the second 
(a)

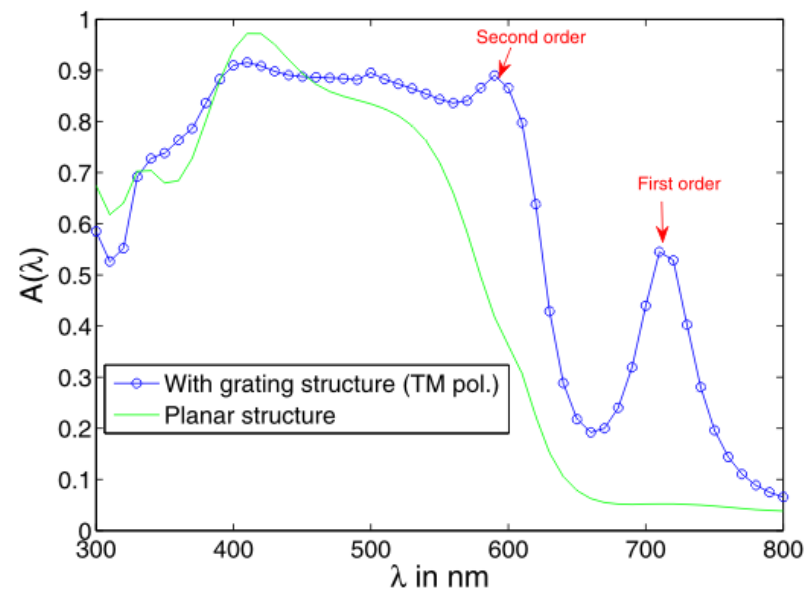

(b)

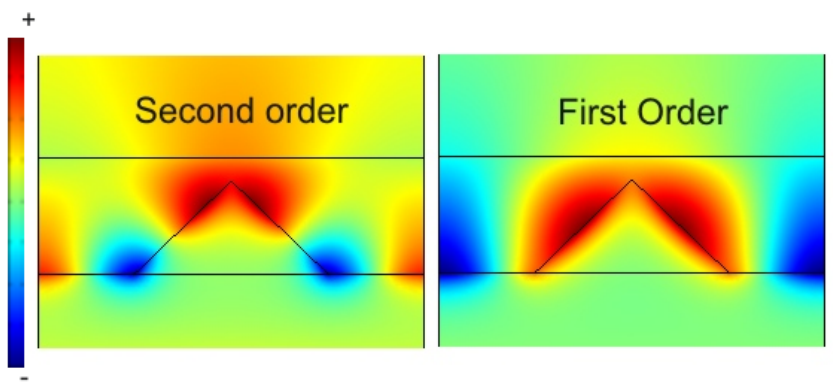

FIG. 7: Employing higher order plasmonic resonances. (a) $A(\lambda)$ of a structure with $\mathrm{P}=500 \mathrm{~nm}$, $\mathrm{T}=150 \mathrm{~nm}, \mathrm{H}=120 \mathrm{~nm}, \mathrm{FF}=0.5$ at normal incidence. (b) The $H_{z}$ magnetic field profile at the first order and at the second order resonance.

order mode is more confined to the metal interface. This makes it more prone to possible exciton quenching.

An optimum structure for wavelengths $<650 \mathrm{~nm}$ and normal incidence which only utilizes the first order bright mode and Fabry-Perot considerations is given in Fig. 8. The grating structure has just a slight influence on the (already good) absorption below 500nm. We note that there is also a significant enhancement for TE polarization, with which no plasmonic resonance can be excited. The integrated absorption efficiency $A_{\text {int }}$ for a planar unpatterned structure is $48 \%$, while for a patterned structure we reach $63.6 \%$ for TM and $56 \%$ for TE polarization. We therefore see a significant $15.6 \%$ increase in $A_{\text {int }}$ for the TM case. 


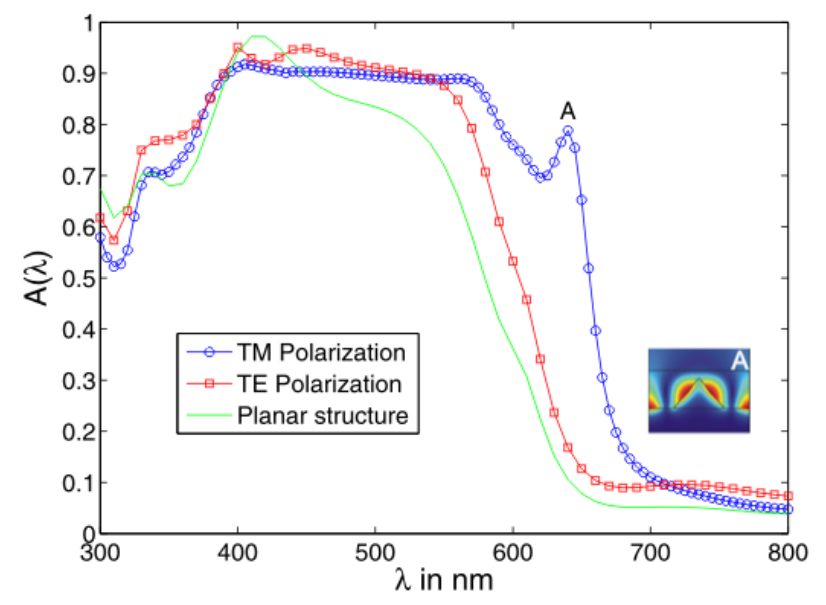

FIG. 8: $A(\lambda)$ of an optimized structure for normal incidence with $\mathrm{T}=150 \mathrm{~nm}, \mathrm{P}=400 \mathrm{~nm}, \mathrm{FF}=0.5$ and $\mathrm{H}=120 \mathrm{~nm}$.

\section{B. Using polymer waveguide modes}

When the polymer thickness is above 200nm, the grating structure can also excite polymer waveguide modes. This provides access to additional resonances to boost the absorption. The use of waveguide excitation has been demonstrated [5, 28], however previous discussions mainly concerned silicon solar cells.

Fig. 9(a) shows the absorption spectrum in such a thicker cell for $\mathrm{T}=250 \mathrm{~nm}, \mathrm{P}=400 \mathrm{~nm}$, $\mathrm{H}=50 \mathrm{~nm}, \mathrm{FF}=0.5$. The origin of the resonances can still be tracked by simple band folding of the waveguide dispersion curve, see Fig. 9(b) where the folded dispersion relation for a flat structure of the same thickness $\mathrm{T}$ is shown (black circles). The line in Fig. 9(b) follows the SPP modes, the other modes have their field mostly in the polymer. The mode that starts around $750 \mathrm{~nm}$, at point A in Fig. 9(a), is the bright first order plasmon mode. The mode starting at $650 \mathrm{~nm}$ and going to point $\mathrm{C}$ stems from a polymer waveguide mode. We observe an anti-crossing interaction (point B) between the waveguide mode and the plasmonic mode, which can happen as they have the same TM polarization. This creates a gap in the dispersion and absorption diagram. It is therefore important to model these interactions, as they might cancel any enhancement in a certain wavelength range. The anti-crossing effect is clear from the field profiles in Fig. 9(c): A shows a SPP mode, B a hybrid SPP-polymer mode, and in $\mathrm{C}$ we have a polymer waveguide mode.

For the structure in Fig. 10 we combine the dark and bright plasmonic modes together 
(a)

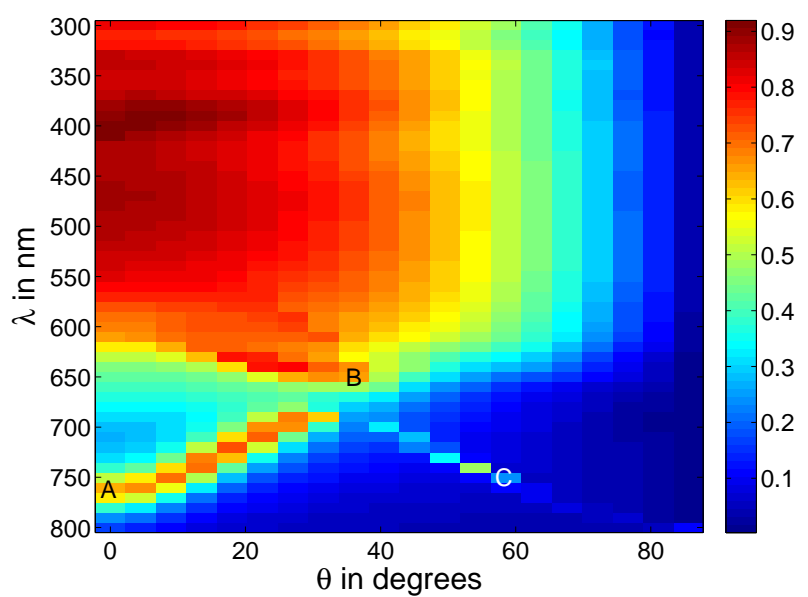

(b)

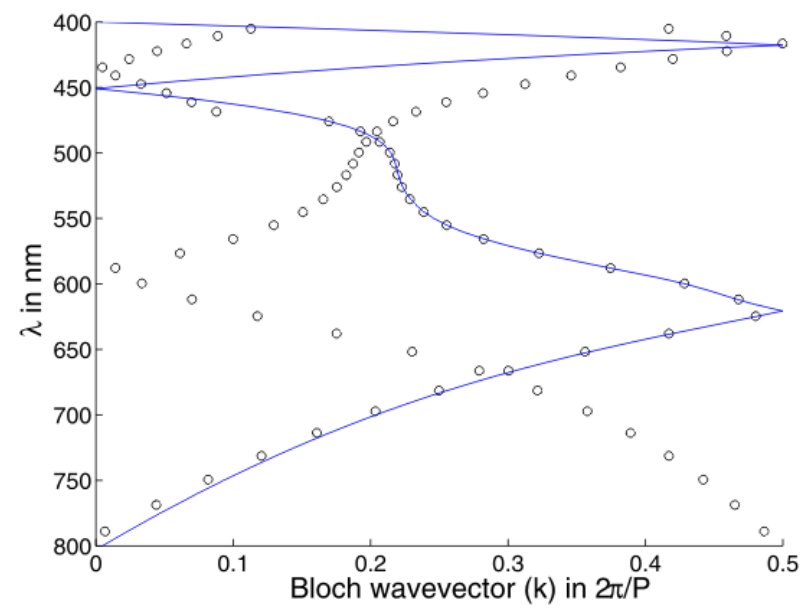

(c)

High
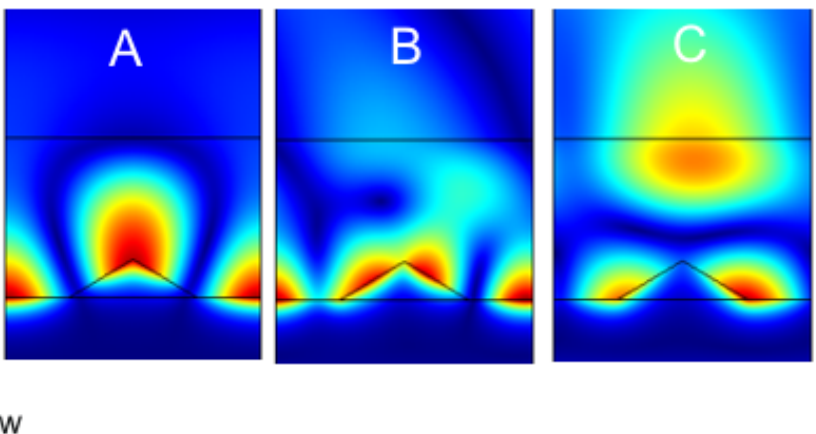

Low

FIG. 9: Solar cell grating system of $\mathrm{T}=250 \mathrm{~nm}, \mathrm{P}=400 \mathrm{~nm}, \mathrm{FF}=0.5$, and $\mathrm{H}=50 \mathrm{~nm}$. (a) Absorption spectrum $A(\lambda)$ in the polymer. (b) Folded dispersion diagram with $400 \mathrm{~nm}$ periodicity. The line is the theoretically calculated SPP mode on polymer-metal interface. (c) Field Profile of the norm of magnetic field at the points indicated in (a). 
with a waveguide mode resonance, for $\theta=13.5^{\circ}$. The absorption spectrum in the wavelength range $600-720 \mathrm{~nm}$ is nicely filled by these three resonances, while the absorption below 550nm remains intact. We obtain an integrated absorption $A_{\text {int }}=71.4 \%$ for TM polarization, and $60.3 \%$ for TE polarization. For a simple planar structure with the same $\mathrm{T}=250 \mathrm{~nm}, A_{\text {int }}$ is only $56 \%$.

The character of the modes is illustrated in Fig. 10(b). The polymer waveguide mode is peak A. The dark mode (peak B) only appears after a change in angle. Note that in this grating the antisymmetric dark mode appears at a smaller wavelength than the bright mode (peak C). We suspect this is due to the thicker polymer layer, leading to a less confined bright mode with smaller energy.

\section{Using strongly localized modes}

Up to this point we showed that the triangle grating structure gives access to a limited number of SPP and waveguide modes. More numerous and highly localized SPP resonances can be used by adding very small subwavelength features to the structure. Various multiperiodic grating structures have been studied before and are found to have multiple resonances with significant absorption capabilities [29-32]. In our case we accomplish this by adding small triangular groove defects with a small period on top of our main triangular grating (inset in Fig. 11(a)). The main grating periodicity is expected to induce band folding on a dispersion relation which now includes additional coupled localized SPP modes due to the defect grooves. We find that this procedure provides multiple resonances with strong absorption enhancements well into the desired enhancement range.

We show a proof of principle in Fig. 11. Fig. 11(a) presents the absorption spectrum of a triangular grating solar cell structure with and without additional groove defects, the inset shows the geometry. We notice that the single plasmonic resonance (peak A) is replaced by four other resonances (C, D, E, F in Fig. 11(b)) which overlap with each other and fill up a large wavelength range. The field profile of these resonances is plotted in Fig. 12. The shoulder (peak B) in Fig. 11(a) is due to Fabry-Perot effects. The field profiles for peaks $\mathrm{C}, \mathrm{D}$ and $\mathrm{E}$ show that the field is heavily concentrated in the defect dips, which is what we would expect from localized surface plasmon resonances of the dips. Resonance $\mathrm{F}$ has its field distribution more spread out, which is expected for a mode associated with the larger 
(a)

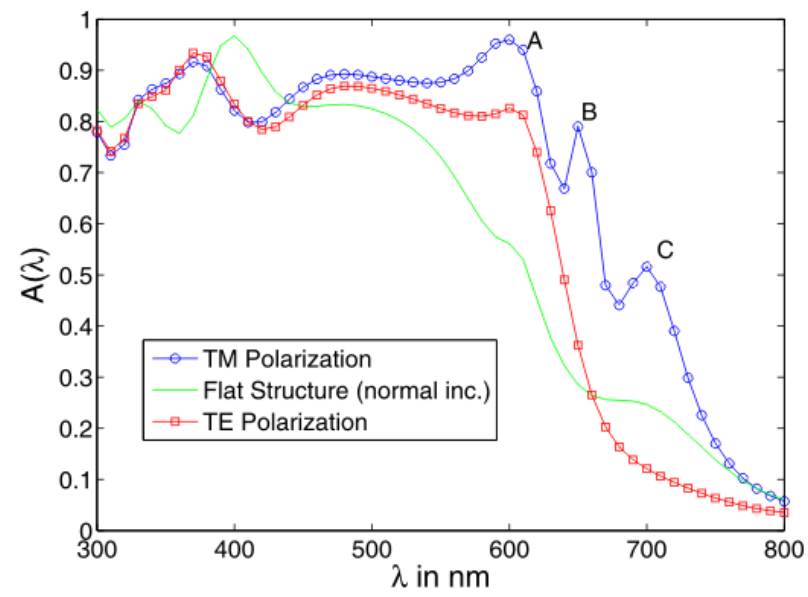

(b)

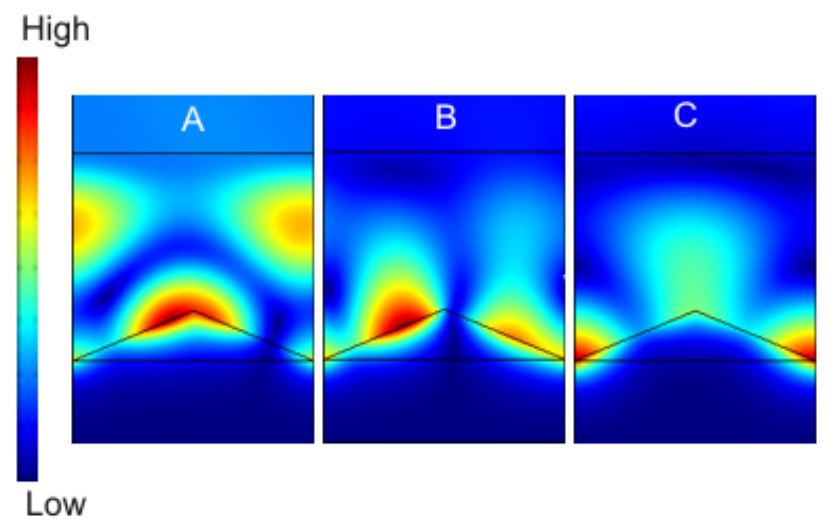

FIG. 10: Employing plasmonic and polymer modes with $\mathrm{T}=250 \mathrm{~nm}, \mathrm{P}=290 \mathrm{~nm}, \mathrm{FF}=1, \mathrm{H}=60 \mathrm{~nm}$. (a) $A(\lambda)$ for the grating cell with $\theta=13.5^{\circ}$, compared with $A(\lambda)$ for a planar structure of $250 \mathrm{~nm}$ polymer with normal incidence. (b) Field profile of the norm of the magnetic field at the three peaks in (a).

triangles (see resonance A), hinting at a link between these resonances.

$A_{\text {int }}$ of the multiperiodic structure reaches $68.7 \%$, which gives us an increase of $20.7 \%$ as compared to the flat structure, and $5.1 \%$ more than the structure with only larger triangles. In addition, the new resonances have a flat dispersion (see Fig. 11(b)), thus their enhancement is angle independent. Further optimization is necessary, but we can already note that a surprisingly large amount of the absorption of the localized modes is in the polymer, and not in the metal, as one might expect at first sight.

The experimental realization of this multiperiodic structure could be done by nanopat- 
(a)

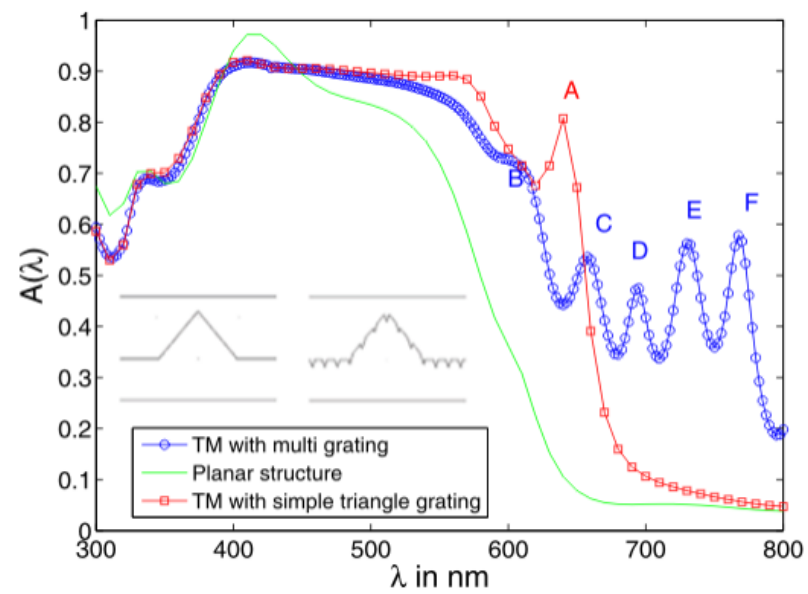

(b)

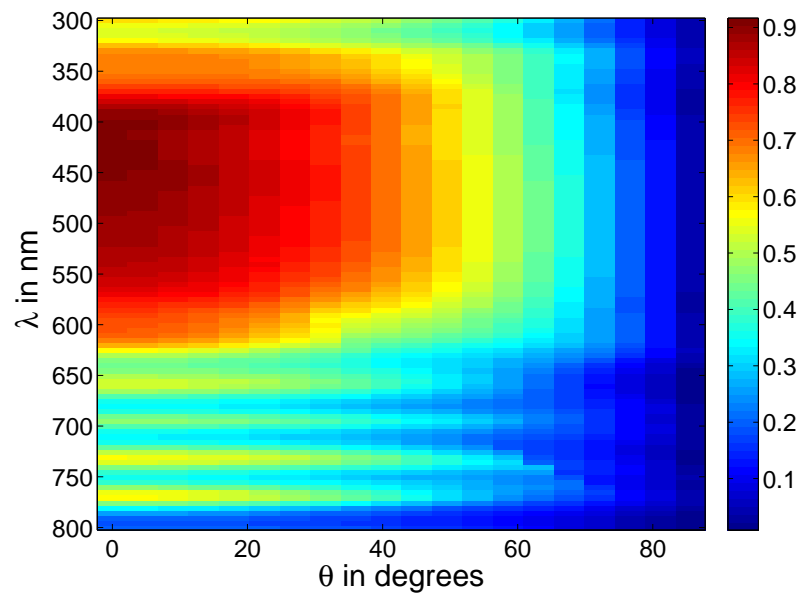

FIG. 11: $A(\lambda)$ for a grating with extra smaller defects (main period 390nm, small period 30nm, main triangle height $120 \mathrm{~nm}$ and $\mathrm{T}=150 \mathrm{~nm}$ ), the defect groove depth is $15 \mathrm{~nm}$ on average, with $2 \mathrm{~nm}$ rounding of the sharp tips. The FF for the main grating is 0.5 and for the defects it is $1 / 3$. (a) $A(\lambda)$ for perpendicular incidence. The plot for the case without additional defects, and for a planar structure with $150 \mathrm{~nm}$ polymer thickness are also provided. The inset shows the geometry. (b) $A(\lambda)$ versus the angle of incidence.

terning, in which the stamp is made using electron beam lithography. However, further investigations on more easily fabricated structures with similar effects are warranted. 


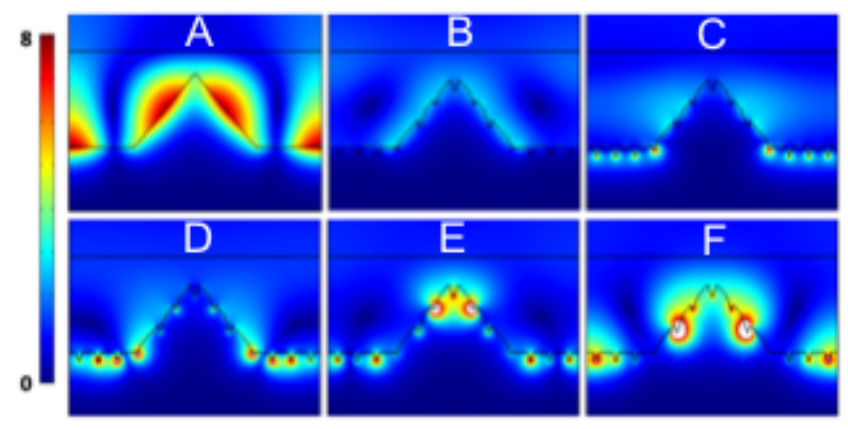

FIG. 12: Norm of magnetic field at the resonances of Fig. 11(a). The field profile is normalized with the same scale shown on the left. The maximum in the color bar corresponds to 8 times the incoming plane wave magnitude. White areas indicate values beyond this range.

\section{Pyramid grating structures}

It is desirable in solar cells to have a polarization independent efficiency. Therefore we study (2D) pyramidal grating structures with rigorous 3D simulations in this section, as opposed to the (1D) triangular gratings before where we only needed 2D simulations. Similar enhancement effects are obtained, but various mode characteristics differ.

Fig. 13 shows results for perpendicular incidence on two solar cell structures with pyramidal gratings of different $F F(T=150 \mathrm{~nm}, \mathrm{H}=120 \mathrm{~nm}, \mathrm{P}=320 \mathrm{~nm})$. It is found that band folding still gives a good prediction in this case. The two peaks in Fig. 13 (for both gratings) stem from a first order crossing around 680nm, see the inset in Fig. 13. Unlike in the $1 \mathrm{D}$ triangular grating case (where $\mathrm{FF}=1$ gives a minimum separation), here the smaller FF gives a smaller separation of the modes. In addition, both modes from the crossing are bright modes.

Fig. 14 presents the absorption spectrum for two different periods ( $T=150 \mathrm{~nm}, H=120 \mathrm{~nm}$, $\mathrm{FF}=1$ ). The first and second order modes appear around the wavelength determined by band folding as before. It can be seen that the dominant peaks in the graph occur at the larger grating periods of $550 \mathrm{~nm}$. The peak around $600 \mathrm{~nm}$ wavelength in this structure is a second order peak. The peak stemming from first order crossings (around 740nm) is considerably weaker than the second order peak. The explanation of these differences is a topic of further study. 


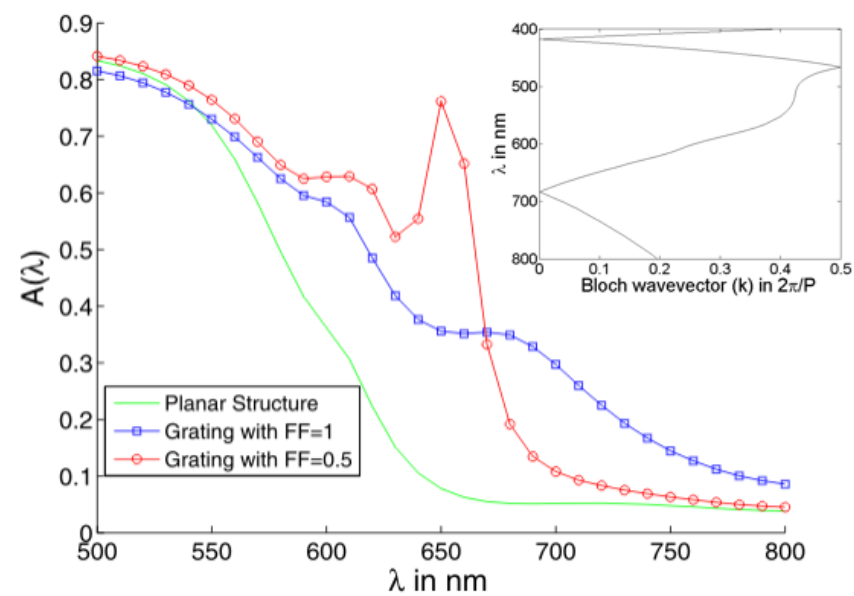

FIG. 13: $A(\lambda)$ at normal incidence for pyramidal gratings (T=150nm, $\mathrm{H}=120 \mathrm{~nm}, \mathrm{P}=320 \mathrm{~nm}$ ) with $\mathrm{FF}=1$ and $\mathrm{FF}=0.5$, respectively. The corresponding planar absorption is also shown. The inset is the folded dispersion curve $(\mathrm{P}=320 \mathrm{~nm})$ of SPP modes on a flat polymer-silver interface.

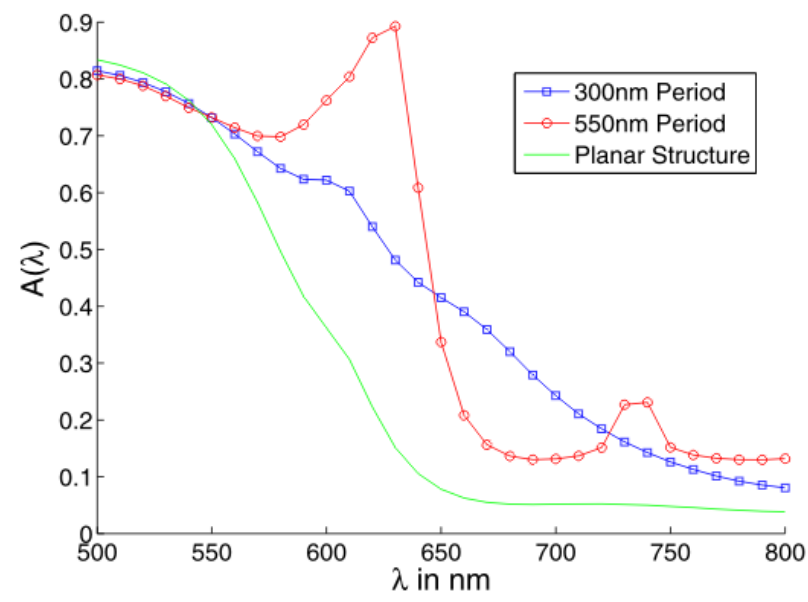

FIG. 14: $A(\lambda)$ at normal incidence in pyramidal gratings for different periods with $\mathrm{FF}=1, \mathrm{~T}=150 \mathrm{~nm}$ and $\mathrm{H}=120 \mathrm{~nm}$. The planar absorption is also shown.

\section{CONCLUSION}

The proposed grating designs combine several physical phenomena (such as SPP, FabryPerot and slab waveguide modes) to boost the absorption capability of a thin polymer film. We focus on enhancing the metallic backreflector with triangular structures in an organic solar cell, however the strategies are applicable to other material systems as well.

We stress the importance of the control of bright and dark modes to obtain an angle- 
dependent optimized structure. The fill factor and the grating height are crucial parameters to this end, as they control aspects such as band flatness, energy difference and sequence of the bright-dark pair.

In addition, we demonstrate the potential of more complicated multiperiodic structures to obtain resonances that fill up a significant wavelength range. This is realized by multiple localized plasmonic resonances with slightly different frequencies, which nonetheless have most of their field in the polymer, and not in the metal.

Finally, 3D simulation results for pyramidal gratings demonstrate the extension of these ideas to polarization independent structures. Band folding e.g. still gives a valid prediction of the resonance locations.

An enhancement of the absorption in the polymer up to 7 times (from $10 \%$ to $70 \%$ ) at a plasmonic resonance is predicted. For the triangular grating we find an increase of $15.6 \%$ for the integrated absorption efficiency (AM1.5G, 300-800nm) compared to a planar structure. The multiperiodic structure gives a larger $20.7 \%$ increase. These results thus show that a major portion of the light absorption is engineered to occur in the active medium.

\section{ACKNOWLEDGEMENTS}

The authors thank B. Niesen for helpful discussions and D. Cheyns for measuring optical constants. This research was supported by the Erasmus Mundus Master in Photonics scholarship program, by the IWT Institute for the Promotion of Innovation by Science and Technology in Flanders via the SBO-project No. 060843 "Polyspec" and by the Interuniversity Attraction Poles program of the Belgian Science Policy Office under Grant No. IAP P6-10 "photonics@be."

[1] G. F. Burkhard, E. T. Hoke, S. R. Scully, and M. D. McGehee, Nano Lett. 9, 40374041 (2009).

[2] S. Cook, A. Furube, R. Katoh, and L. Han, Chem. Phys. Lett. 478, 33-36 (2009).

[3] P. E. Shaw, A. Ruseckas, and D. W. Samuel, Adv. Mater. 20, 3516-3520 (2008).

[4] K. Petritsch, Ph.D. thesis, Universitt Graz, Austria (2008)

[5] H. Atwater and A. Polman, Nature Materials 9, 205213 (2010).

[6] H. Shen, P. Bienstman, and B. Maes, J. Appl. Phys. 106, 073109 (2009). 
[7] I. M. Pryce, D. D. Koleske, A. J. Fischer, and H. A. Atwater, Appl. Phys. Lett. 96, 153501 (2010).

[8] K. R. Catchpole, and A. Polman, Opt. Express 26, 21793 (2008).

[9] K. R. Catchpole, and A. Polman, Appl. Phys. Lett. 93, 191113 (2008).

[10] K. Tvingstedt, N. Persson, O. Ingans, A. Rahachou, and I. V. Zozoulenko, Appl. Phys. Lett., 91, 113514 (2007).

[11] A. P. Kulkarni, K. M. Noone, K. Munechika, S. R. Guyer, and D. S. Ginger, Nano Lett. 10, 15011505 (2010).

[12] W. Bai, Q. Gan, F. Bartoli, J. Zhang, L. Cai, Y. Huang, and G. Song, Opt. Lett. 34, 3725 (2009).

[13] R. Dewan, R. N. M. Marinkovic, S. Phadke, A. Salleo, and D. Knipp, Opt. Express 17, 23058 (2009).

[14] R. A. Pala, J. White, E. Barnard, J. Liu, and M. L. Brongersma, Adv. Mater. 21, 3504 (2009).

[15] W. Wang, S. Wu, K. Reinhardt, Y. Lu, and S. Chen, Nano Lett. 10, 2012-2018 (2010).

[16] L. S. Roman, O. ingans, T. Granlund, T. Nyberg, and M. Svensson, Adv. Mater. 12, 189 (2000).

[17] C. Cocoyer, L.Rocha, L. Sicot, B. Geoffroy, R. de Bettignies, C. Sentein, C. FioriniDebuisschert, and P. Raimond, Appl. Phys. Lett. 88, 133108 (2006).

[18] S. -I. Na, S. -S. Kim, J. Jo, S. -H. Oh, J. Kim, and D. -Y. Kim, Adv. Func. Mater. 18, 3956 (2008).

[19] C. Chen, N. Verellen, K. Lodewijks, L. Lagae, G. Maes, G. Borghs, and P. Van Dorpe, J. Appl. Phys. 108, 034319 (2010).

[20] G. Veronis, R. W. Dutton, and S. Fan, J. Appl. Phys. 97, 093104 (2005).

[21] P. D. Cunningham and L.M. Hayden, J. Phys. Chem. C 112, 7928 (2008).

[22] J. D. Joannopoulos, S. G. Johnson, J. N. Winn, and R. D. Meade, Molding the flow of light, 2nd edition (Princeton University Press, 2008).

[23] W. L. Barnes, T. W. Preist, S. C. Kitson, and J. R. Sambles, Phys. Rev. B 54, 6227 (1996).

[24] I. R. Hooper and J. R. Sambles, Phys. Rev. B 65, 165432 (2002).

[25] I. R. Hooper and J. R. Sambles, Phys. Rev. B 66, 205408 (2002).

[26] V. Agranovich and A. Malshukov, Chem. Phys. Lett. 43, 221 (1976).

[27] G. Vecchi, V. Giannini, and J. G. Rivas, Phys. Rev. B 80, 201401 (2009). 
[28] V.E. Ferry, L.A. Sweatlock, D.Pacifici, and Harry A. Atwater, Nano Lett. 8, 4391-4397 (2008).

[29] W.C. Tan, J. R. Sambles, and T. W. Preist, Phys. Rev. B 61, 13177 (2000)

[30] A. P. Hibbins, J. R. Sambles, and C. R. Lawrence, Appl. Phys. Lett. 80, 2410 (2002)

[31] M. J. Lockyear, A. P. Hibbins, J. R. Sambles, and C. R. Lawrence, Appl. Phys. Lett. 83, 806 (2003)

[32] M. J. Lockyear, A. P. Hibbins, J. R. Sambles, and C. R. Lawrence, Appl. Phys. Lett. 86, $184103(2005)$ 